\title{
Impact of aspiration catheter size on first-pass effect in the combined use of contact aspiration and stent retriever technique
}

\author{
Carlos Pérez-García (10 , ${ }^{1}$ Christian Maegerlein, ${ }^{2}$ Santiago Rosati, ${ }^{1}$ \\ Charlotte Rüther, ${ }^{2}$ Carlos Gómez-Escalonilla, ${ }^{3}$ Claus Zimmer, ${ }^{2}$ Juan Arrazola, ${ }^{4}$ \\ Maria Teresa Berndt, ${ }^{2}$ Manuel Moreu ${ }^{1}$
}

To cite: Pérez-García C, Maegerlein C, Rosati S, et al. Impact of aspiration catheter size on first-pass effect in the combined use of contact aspiration and stent retriever technique. Stroke \& Vascular Neurology 2021;0. doi:10.1136/ svn-2020-000833

Received 26 December 2020 Revised 27 February 2021 Accepted 5 March 2021
Check for updates

(C) Author(s) (or their employer(s)) 2021. Re-use permitted under CC BY-NC. No commercial re-use. See rights and permissions. Published by BMJ.

${ }^{1}$ Interventional Neuroradiology, Hospital Clinico Universitario San Carlos, Madrid, Spain

${ }^{2}$ Department of Diagnostic and Interventional Neuroradiology, Klinikum rechts der Isar der Technischen Universitat Munchen, Munchen, Bayern, Germany

${ }^{3}$ Neurology, Hospital Clinico Universitario San Carlos, Madrid, Spain

${ }^{4}$ Radiology, Hospital Clinico Universitario San Carlos, Madrid, Spain

Correspondence to Dr Carlos Pérez-García; cperezgarcia.med@gmail.com

\section{ABSTRACT}

Background and purpose The first-pass effect (FPE), defined as a first-pass Expanded Treatment in Cerebral Ischaemia (eTICl) 2c/3 reperfusion, has emerged as a key metric of efficacy in mechanical thrombectomy (MT) for acute ischaemic stroke. The proximal balloon occlusion together with direct thrombus aspiration during stent retriever thrombectomy (PROTECT)-PLUS technique consists in the use of a balloon guide catheter and a combined MT approach involving contact aspiration and a stent retriever. The aim of this study is to assess the effectiveness and safety of the PROTECT-PLUS technique using distal aspiration catheters (DACs) with different inner diameters by comparing the large-bore DAC Catalyst 7 versus the use of medium-bore DACs.

Methods Retrospective analysis of a prospectively maintained database of patients treated with PROTECTPLUS using Catalyst 7, Catalyst 6 or Catalyst 5 with an occlusion of either the terminal carotid artery or the M1 or M2 segments of the middle cerebral artery from 2018 to 2020 in two comprehensive stroke centres. Baseline characteristics and procedural, safety and clinical outcomes were compared between groups. Multivariable logistic regression analysis was performed in order to find independent predictors of FPE.

Results We identified 238 consecutive patients treated with PROTECT-PLUS as front-line approach using Catalyst $7(n=86)$, Catalyst $6(n=78)$ and Catalyst $5(n=76)$. The rate of FPE was higher with Catalyst $7(54 \%)$ than Catalyst $6(33 \%, p=0.009)$ and Catalyst $5(32 \%, p=0.005)$, in addition to higher final eTICI $2 \mathrm{c} / 3$ reperfusion rates, shorter procedural times, lower need of rescue therapy and fewer procedure-related complications. After multivariable analysis the sole independent factor associated to FPE was the use of Catalyst 7 (OR 2.34; $95 \% \mathrm{Cl} 1.19$ to 4.58; $p=0.014$ ).

Conclusion Further development of combined MT by incorporating larger-bore aspiration catheters is associated with higher reperfusion rates, shorter procedure times, and lower need of rescue therapy while reducing the complication rates.

\section{INTRODUCTION}

After landmark randomised controlled trials showed that mechanical thrombectomy (MT) with stent retrievers, with or without intravenous administration of fibrinolysis, is the treatment of choice in patients with intracranial anterior circulation large vessel occlusion (LVO), ${ }^{1}$ procedural and technological efforts have been made to continuously enhance the technical aspects of MT.

Successful reperfusion has traditionally been defined as Modified Treatment in Cerebral Ischaemia (mTICI) score $2 \mathrm{~b} / 3$, meaning reperfusion of at least half of the affected territory. However, it has become increasingly evident that the clinical outcome of patients with only half of the middle cerebral artery (MCA) territory reperfused is not as good as those where Expanded Treatment in Cerebral Ischaemia (eTICI) score $2 \mathrm{c} / 3$ is achieved, therefore complete/near complete reperfusion should be the aim. ${ }^{2}{ }^{3}$ Furthermore, the first-pass effect (FPE), defined as a single-pass eTICI 2c/3 reperfusion with no use of additional passes/devices, ${ }^{4}$ has been proven to be an independent predictor of good outcome, probably as fast complete reperfusion avoids multiple thrombectomy passes with the added risk of endothelial injury and emboli to undesired territories. ${ }^{5}$

A modification of the technique that has become widely accepted in recent years is the use of balloon guide catheters (BGCs), ${ }^{6-10}$ and multiple techniques for faster and more efficient thrombus extraction have evolved, including front-line aspiration ${ }^{11}{ }^{12}$ and combined approaches. ${ }^{1314}$ Several advantages of the latter have been described, including pinching the thrombus between the stent retriever and the distal aspiration catheter (DAC), allowing the clot to be held more effectively during removal, ${ }^{13}$ while reducing the contact surface between the stent retriever and the vessel wall, thus potentially lowering the risk of vascular injury. 
Recently, the authors suggested the use of BGC along with combined MT technique, withdrawing the stent retriever device inside the DAC (proximal balloon occlusion together with direct thrombus aspiration during stent retriever thrombectomy (PROTECT) technique)..$^{85}$ Maus $e t a l^{13}$ described a modification of the combined MT approach, the stent retriever assisted vacuumlocked extraction (SAVE) technique, consisting in the deployment of the stent retriever over the occlusion, the advancement of the DAC to the face of the clot and then withdrawing both the stent retriever and the DAC as a unit. Subsequently, as a further development, the authors introduced the PROTECT-PLUS technique, a combination of SAVE and PROTECT techniques. ${ }^{16}$

The use of DACs with various inner diameters has already been assessed with the direct aspiration first-pass technique (ADAPT) ${ }^{17}{ }^{18}$; however, the performance of different size DACs has not been evaluated in combined MT approaches. Our aim in this study was to compare the effectiveness and safety of the PROTECT-PLUS technique using the large-bore DAC AXS Catalyst 7 (Stryker, Kalamazoo, Michigan, USA) versus the use of the medium-bore DACs AXS Catalyst 6 and AXS Catalyst 5 (Stryker).

\section{METHODS}

\section{Study design and patient selection}

A retrospective analysis of a prospective database of patients undergoing MT for acute ischaemic stroke was conducted at two comprehensive stroke centres. All consecutive patients who had anterior circulation acute ischaemic stroke treated with PROTECT-PLUS as the front-line approach were identified from January 2018 to March 2020. Patients were eligible for MT with PROTECT-PLUS if the CT angiography confirmed the LVO of the terminus internal carotid artery (ICA) or M1/M2 segments of the MCA. Parenchymal infarction was limited to an ASPECTS of $>5$. No age or perfusion selection was applied.

\section{Device description}

The proximal BGC used in all procedures was Flowgate2 (Stryker). The microcatheter employed was a $150 \mathrm{~cm}$ long 0.021 " Trevo 18 (Stryker). The stent retrievers used were Aperio (Acandis, Pforzheim, Germany), Catch (Balt, Montmorency, France), Embotrap II (Cerenovus, Galway, Ireland), pREset (Phenox, Bochum, Germany) and Trevo XP Provue (Stryker). The $132 \mathrm{~cm}-$ long DACs employed were the large-bore 0.068 " distal inner diameter Catalyst 7, medium-bore 0.060" distal inner diameter Catalyst 6 and medium-bore 0.058" distal inner diameter Catalyst 5 .

The choice of the size of the DAC and the type of stent retriever in each case was primarily driven by availability in the department and ultimately to the criterion of the neurointerventionist.

\section{Interventional protocol}

All interventions were performed by seven experienced interventional neuroradiologists, and both centres used
A

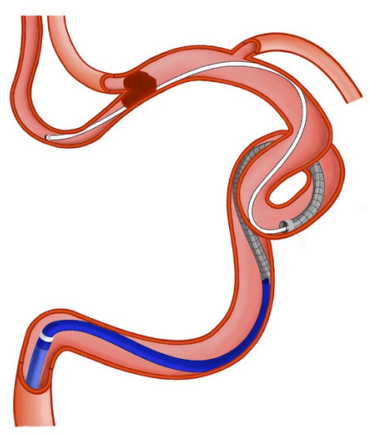

C

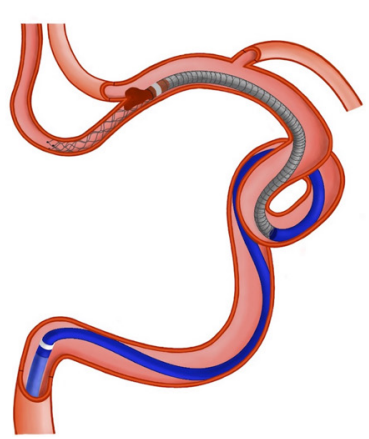

$\mathrm{D}$
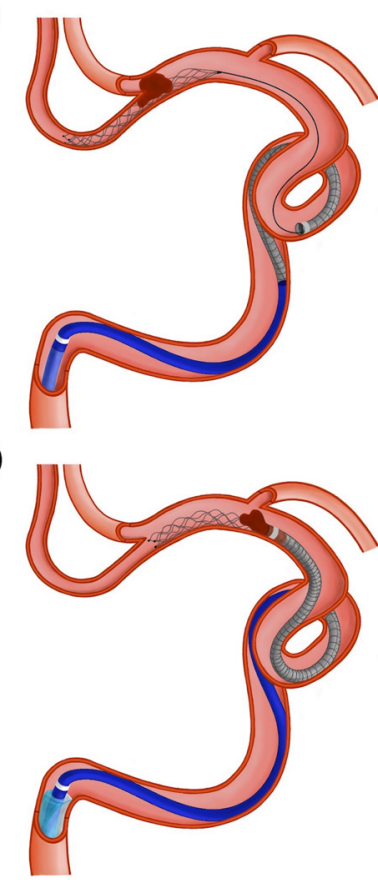

Figure 1 Illustrations depicting the PROTECT-PLUS technique. (A) A 0.021 " microcatheter is initially navigated distal to the thrombus, while the DAC remains in the cavernous ICA. (B) The stent retriever is deployed with the proximal third across the occlusion site and the microcatheter is completely removed. (C) The DAC is advanced into the face of the clot while a slight traction is applied to the stent retriever pusher wire to provide an easier navigation. Aspiration through the DAC is initiated before reaching the clot. (D) The balloon of the BGC is inflated and both the stent retriever and the DAC are withdrawn as a unit under constant aspiration both from the BGC and DAC. BGC, balloon guide catheter; DAC, distal aspiration catheter; ICA, internal carotid artery; PROTECT, proximal balloon occlusion together with direct thrombus aspiration during stent retriever thrombectomy.

the same main devices and the following MT technique. Through a femoral access, the affected side cervical ICA was catheterised using a BGC. The PROTECT-PLUS technique (figure 1) consists in the navigation of the microcatheter under roadmap guidance over a 0.014 " microwire distal to the occlusion, using the DAC as a support, usually with no need to advance it primarily beyond the origin of the ophthalmic artery. The stent retriever is deployed using the active push and fluff deployment technique, ${ }^{19}$ with the proximal third across the thrombus. The microcatheter is removed completely to increase the aspiration volume of the DAC. In order to advance the DAC to the face of the thrombus (indicated by the presence of a vacuum on the DAC), a slight traction is applied to the stent retriever delivery wire to straighten the vessel curves, providing easier navigation of the DAC. Before reaching the clot with the DAC, aspiration is applied using an electric pump. Finally, a torque device is applied to the pusher wire; the balloon of the BGC is inflated; and the stent retriever and the DAC are slowly retrieved 
outright back as a unit under constant aspiration both from the BGC and the DAC.

\section{Image and patient analysis}

Preintervention and postintervention CT scans, in addition to the procedure angiograms, were independently reviewed by two interventional neuroradiologists. This image review included the initial CT ASPECTS, the occlusion site (terminus ICA, MCA M1/M2 segments and tandem occlusions), the arc type (types I-III), ${ }^{20}$ cervical vessel tortuosity (straight, tortuosity, coiling and kinking), ${ }^{21}$ intracranial vessel tortuosity (types IA, IB, II, III and IV), ${ }^{22}$ the first-pass and final eTICI reperfusion scores, the presence of emboli to new territory (ENT) after the MT attempt and haemorrhagic complications in the postintervention CT scan.

The patient baseline characteristics, secondary procedural findings and clinical outcomes were collected from a neurointerventional prospective register together with the neurology department. This included the TOAST classification of the stroke (atherothrombotic, cardioembolic, other or undetermined), ${ }^{23}$ the time from onset to groin puncture and groin puncture to reperfusion, the devices used, the number of MT attempts, the need of rescue therapy (use of other DAC or technique after failure of the front-line approach) and the Modified Rankin Scale (mRS) score at 90 days through face-to-face assessments or standardised telephone interviews.

\section{Outcome}

The main outcome of our study was the rate of FPE. Secondary efficacy endpoints included the end-ofprocedure eTICI reperfusion score, the overall number of MT attempts, time from femoral puncture to reperfusion and the need of rescue therapy. The safety outcome endpoints recorded were the occurrence of ENT, iatrogenic vessel dissection, subarachnoid haemorrhage and symptomatic intracranial haemorrhage (sICH). The clinical outcomes included the rate of mortality and good outcome (mRS score 0-2) at 90 days.

\section{Statistical analysis}

Statistical analysis was performed using IBM SPSS Statistics V.25. Patients were divided in three groups according to the DAC used (Catalyst 5, 6 or 7). We compared the baseline characteristics and procedural, safety and clinical outcomes between the Catalyst 7 group and the Catalyst 6 and Catalyst 5 groups each separately.

For comparing categorical variables between groups, we used $\chi^{2}$ test or Fisher exact test, and when comparing quantitative variables, Mann-Whitney U test was employed. Multivariable logistic regression analysis was performed in order to identify independent variables associated with first-pass eTICI 2c or 3 reperfusions. First, we performed a univariable logistic regression analysis with the variables potentially associated with vessel recanalisation: age, initial CT ASPECTS, baseline NIHSS score, time from onset to groin, use of intravenous thrombolysis, occlusion sites, clot origins, the stroke centre, the stent retriever employed and DACs employed. To maximise sensitivity, those variables with a univariable association of $\mathrm{p}<0.20$ were included into a multivariable logistic regression model. Quantitative variables were not dichotomised but adjusted per 1 year increase (age), 1-point increase (ASPECTS and NIHSS score) and 1 min increase (time from onset to groin). In addition, to ensure that multicollinearity did not impact the results, variance inflation factor was tested on all independent variables. Associations are presented as ORs with corresponding 95\% CIs.

\section{RESULTS}

\section{Patient inclusion}

A total of 238 consecutive patients (mean age $70.4 \pm 12.9$ years, female:male ratio 140:98) underwent MT for anterior circulation LVO acute ischaemic stroke using PROTECT-PLUS technique as the front-line approach from January 2018 to March 2020 in the two comprehensive stroke centres which participated in the present study. During the period of our study, all medium-bore and large-bore DACs were successfully navigated to reach the face of the thrombus in all patients treated with the intention to apply PROTECT-PLUS. The DACs used during the study period were Catalyst $7(\mathrm{n}=86,36.1 \%)$, Catalyst $6(\mathrm{n}=78,32.8 \%)$ and Catalyst $5(\mathrm{n}=76,31.9 \%)$.

\section{Baseline characteristics}

There were no statistical differences between groups regarding demographics, ASPECTS on initial CT, clinical severity using the NIHSS score, the use of intravenous thrombolysis, time from onset to femoral puncture or the use of general anaesthesia (table 1). Groups were also comparable regarding the occlusion site (including tandem occlusions) and the clot aetiology, with no differences in terms of the arc type and the cervical or intracranial vessel tortuosity, in addition to a balanced distribution in the use of the five stent retrievers.

\section{Outcomes}

The procedural, safety and clinical outcomes are shown in table 2. After the first pass, the Catalyst 7 group achieved a significantly higher rate of eTICI $2 c / 3$ reperfusion $(53.6 \%)$ than both the Catalyst $6(33.3 \%, \mathrm{p}=0.009)$ and Catalyst $5(31.6 \%, \mathrm{p}=0.005)$ groups, in addition to a higher eTICI $2 c / 3$ reperfusion rate at the end of the procedure, $70.2 \%$ in the Catalyst 7 group, $50 \%$ using Catalyst $6(\mathrm{p}=0.008)$ and $53.9 \%$ with Catalyst $5(\mathrm{p}=0.034)$ (figure 2).

Overall, in 63 patients $(26.5 \%)$, the MT approach was changed during the procedure after failure of the frontline PROTECT-PLUS technique, and the most frequently employed rescue therapy was the use of a stent retriever alone $(n=42,66.7 \%)$, followed by contact aspiration alone $(n=14,22.2 \%)$, balloon angioplasty $(n=3,4.8 \%)$, balloon angioplasty+permanent intracranial stent placement $(n=2,3.2 \%)$ and intravenous glycoprotein IIb/ IIIa inhibitors alone $(n=2,3.2 \%)$. In cases where balloon 
Table 1 Baseline characteristics, occlusion site, clot aetiology and stent retrievers used between groups

\begin{tabular}{|c|c|c|c|c|c|}
\hline \multirow[b]{2}{*}{ Characteristics } & \multirow[b]{2}{*}{$\begin{array}{l}\text { Cat } 5 \\
(n=76)\end{array}$} & \multirow[b]{2}{*}{$\begin{array}{l}\text { Cat } 6 \\
(n=78)\end{array}$} & \multirow[b]{2}{*}{$\begin{array}{l}\text { Cat } 7 \\
(n=84) \\
\end{array}$} & \multicolumn{2}{|l|}{$P$ value } \\
\hline & & & & $\begin{array}{l}\text { Cat } 5 \text { versus } \\
\text { Cat } 7\end{array}$ & $\begin{array}{l}\text { Cat } 6 \text { versus } \\
\text { Cat } 7\end{array}$ \\
\hline Age (years) & 78 (71.0-83.3) & $73.5(62.8-84.0)$ & $74(63-82)$ & 0.132 & 0.609 \\
\hline Female sex & $48(63.2)$ & $43(55.1)$ & $49(58.3)$ & 0.533 & 0.681 \\
\hline ASPECTS & $8(7-9)$ & $9(7-10)$ & $8(7-9)$ & 0.339 & 0.094 \\
\hline NIHSS score & $18.5(12-23)$ & $17.5(10-20.8)$ & $16(12-20)$ & 0.129 & 0.667 \\
\hline Intravenous tPA & $23(30.3)$ & $36(46.2)$ & $34(40.5)$ & 0.178 & 0.466 \\
\hline Time onset-groin (min) & $210(163-325)$ & $195(155-345)$ & $230(162-324)$ & 0.564 & 0.651 \\
\hline General anaesthesia & $28(36.8)$ & $43(55.1)$ & $40(47.6)$ & 0.168 & 0.726 \\
\hline Tandem occlusion & $11(14.5)$ & $13(16.7)$ & $13(15.5)$ & 0.859 & 0.837 \\
\hline Occlusion site & & & & 0.304 & 0.195 \\
\hline Carotid terminus & $20(26.3)$ & $22(28.2)$ & $30(35.7)$ & & \\
\hline MCA M1 & $43(56.6)$ & $40(51.3)$ & 45 (53.6) & & \\
\hline MCA M2 & $13(17.1)$ & $16(20.5)$ & $9(10.7)$ & & \\
\hline Clot origin & & & & 0.070 & 0.319 \\
\hline Atherothrombotic & $5(6.6)$ & 14 (17.9) & $10(11.9)$ & & \\
\hline Cardioembolic & $54(71.1)$ & $42(53.8)$ & 48 (57.1) & & \\
\hline Other & $0(0)$ & $1(1.3)$ & $5(6)$ & & \\
\hline Undetermined & $17(22.4)$ & $21(26.9)$ & $21(25)$ & & \\
\hline Arch type & & & & 0.728 & 0.064 \\
\hline Type I & $17(22.4)$ & $23(29.5)$ & $23(22.4)$ & & \\
\hline Type II & 44 (57.9) & $51(65.4)$ & 47 (57.9) & & \\
\hline Type III & 15 (19.7) & $4(5.1)$ & 14 (17.9) & & \\
\hline Cervical tortuosity & & & & 0.764 & 0.143 \\
\hline Straight-tortuosity & $47(61.8)$ & 55 (70.5) & $50(59.5)$ & & \\
\hline Coiling-kinking & 29 (38.2) & $23(29.5)$ & $34(40.5)$ & & \\
\hline Intracranial tortuosity & & & & 0.094 & 0.140 \\
\hline Types IA and IB & $48(63.2)$ & $48(61.5)$ & $42(50)$ & & \\
\hline Types II, III and IV & $28(36.8)$ & $30(38.5)$ & $42(50)$ & & \\
\hline Stent retrievers used & & & & 0.157 & 0.430 \\
\hline Aperio & $14(18.4)$ & $10(12.8)$ & $13(15.5)$ & & \\
\hline Catch & $10(13.2)$ & $7(9.0)$ & $2(2.4)$ & & \\
\hline Embotrap & $6(7.9)$ & $8(10.3)$ & $8(9.5)$ & & \\
\hline pREset & 25 (32.9) & $34(43.6)$ & 36 (42.9) & & \\
\hline Trevo & 21 (27.6) & $19(24.4)$ & $25(29.8)$ & & \\
\hline
\end{tabular}

Values are presented as number (\%) or median (IQR).

ASPECTS, Alberta Stroke Program Early CT Score; Cat, Catalyst; MCA, middle cerebral artery; NIHSS, National Institutes of Health Stroke Scale; tPA, tissue-type plasminogen activator.

angioplasty \pm intracranial stenting was performed, glycoprotein IIb/IIIa inhibitors had previously been administered. The need of rescue therapy was significantly lower in the Catalyst 7 group $(\mathrm{n}=13,15.5 \%)$ compared with the Catalyst $6(\mathrm{n}=25,32.1 \%$; $\mathrm{p}=0.013)$ and Catalyst $5(\mathrm{n}=25,32.9 \% ; \mathrm{p}=0.010)$ groups, in addition to a lower overall number of thrombectomy passes with the use of Catalyst 7. Time from groin puncture to reperfusion was shorter with Catalyst seven than Catalyst 5 (30.5 min vs
38.5 min, $\mathrm{p}=0.044)$, whereas compared with the Catalyst 6 group (36.5 min) it did not reach statistical significance $(\mathrm{p}=0.154)$.

In terms of safety outcomes, we found a lower incidence of subarachnoid haemorrhage in the Catalyst 7 cohort when compared with the Catalyst 5 group $(1.3 \%$ vs $7.9 \%$, $\mathrm{p}=0.038$ ). No differences were found between the groups regarding the rate of sICH, the occurrence of ENT and iatrogenic vessel dissection. Nevertheless, considering the 
Table 2 Procedural, safety and clinical outcomes between groups

\begin{tabular}{|c|c|c|c|c|c|}
\hline \multirow[b]{2}{*}{ Characteristics } & \multirow[b]{2}{*}{ Cat $5(n=76)$} & \multirow[b]{2}{*}{ Cat $6(n=78)$} & \multirow[b]{2}{*}{ Cat $7(n=84)$} & \multicolumn{2}{|l|}{$P$ value } \\
\hline & & & & Cat 5 versus Cat 7 & Cat 6 versus Cat 7 \\
\hline \multicolumn{6}{|l|}{ Procedural outcomes } \\
\hline First-pass effect & $24(31.6)$ & $26(33.3)$ & $45(53.6)$ & 0.005 & 0.009 \\
\hline Final eTICI 2c/3 & $41(53.9)$ & $39(50)$ & $59(70.2)$ & 0.034 & 0.008 \\
\hline Final eTICl 2b/2c/3 & $58(76.3)$ & $73(93.4)$ & $79(94)$ & 0.001 & 0.904 \\
\hline Number of MT attempts & $2(1-4)$ & $2(1-3)$ & $1(1-2.3)$ & 0.009 & 0.014 \\
\hline Need of rescue therapy & 25 (32.9) & $25(32.1)$ & $13(15.5)$ & 0.010 & 0.013 \\
\hline Time groin-reperfusion & $38.5(25.8-64)$ & $36.5(20-67)$ & $30.5(21-48.8)$ & 0.044 & 0.154 \\
\hline \multicolumn{6}{|l|}{ Safety outcomes } \\
\hline $\mathrm{SAH}$ & $6(7.9)$ & $3(3.8)$ & $1(1.2)$ & 0.038 & 0.276 \\
\hline $\mathrm{sICH}$ & $2(2.6)$ & $4(5.1)$ & $1(1.2)$ & 0.502 & 0.148 \\
\hline ENT & $4(5.3)$ & $4(5.1)$ & $1(1.2)$ & 0.139 & 0.148 \\
\hline latrogenic dissection & $1(1.3)$ & $2(2.6)$ & $2(2.4)$ & 0.620 & 0.940 \\
\hline Overall complications & $13(17.1)$ & $13(16.7)$ & $5(5.9)$ & 0.026 & 0.030 \\
\hline \multicolumn{6}{|l|}{ Clinical outcomes } \\
\hline Mortality at 90 days & $18(23.7)$ & $14(17.9)$ & 15 (17.9) & 0.363 & 0.988 \\
\hline $\begin{array}{l}\mathrm{mRS} \text { score of } 0-2 \text { at } 90 \\
\text { days }\end{array}$ & $35(46.1)$ & $39(50)$ & $46(54.8)$ & 0.271 & 0.544 \\
\hline
\end{tabular}

Values are presented as number (\%) or median (IQR).

$P$-values numbers marked in bold indicate statistically significant differences $(<0.05)$.

Cat, Catalyst; ENT, emboli to new territory; eTICI, expanded thrombolysis in cerebral ischaemia; mRS, Modified Rankin Scale; MT, mechanical thrombectomy; $\mathrm{SAH}$, subarachnoid haemorrhage; $\mathrm{sICH}$, symptomatic intracranial haemorrhage.

total number of procedure-related complications, the use of Catalyst 7 was associated with fewer overall complications $(\mathrm{n}=5,5.9 \%)$ compared with Catalyst $6(\mathrm{n}=13,16.7 \%$; $\mathrm{p}=0.030)$ and Catalyst $5(\mathrm{n}=13,17.1 \% ; \mathrm{p}=0.026)$.

The clinical outcomes were comparable between groups with an overall mortality at 3 months of $19.7 \%$ $(\mathrm{n}=47)$ and a trend towards a higher mRS score of $0-2$

Final expanded Treatment in Cerebral Ischaemia score

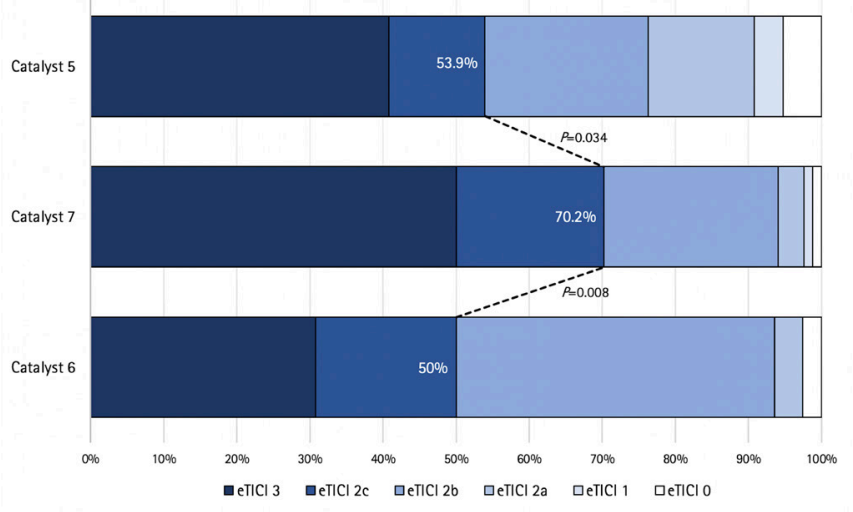

Figure 2 Distribution of final eTICl score comparing the PROTECT-PLUS technique with Catalyst 7 versus the PROTECT-PLUS technique with Catalysts 5 and 6 . eTICl, expanded treatment in cerebral ischaemia; PROTECT, proximal balloon occlusion together with direct thrombus aspiration during stent retriever thrombectomy. at 90 days from the smallest to the largest DAC, $46.1 \%$ $(\mathrm{n}=35)$ with Catalyst 5, 50\% (n=39) using Catalyst $6 \%$ and $54.8 \%(\mathrm{n}=46)$ with Catalyst 7 . Among patients in whom FPE was achieved, $66.7 \%$ reached functional independence at 3 months; however, this rate decreased to $40.1 \%$ when complete reperfusion after the first pass was not achieved, with statistically significant differences $(\mathrm{p}<0.001)$.

\section{Predictors of FPE}

To find independent predictors associated with FPE, variables with a $p$ value of $<0.20$ in the univariable logistic regression analysis (initial CT ASPECTS, M2 occlusion and use of Aperio, Embotrap, Trevo, Catalyst 5 and Catalyst 7) were included in the multivariable logistic regression analysis (table 3), where the sole independent factor associated with FPE was the use of Catalyst $7(\mathrm{p}=0.014)$ with an OR of 2.34 (95\% CI 1.19 to 4.58$)$.

\section{DISCUSSION}

Overall, our findings suggest that the use of a larger-bore DAC may lead to improved efficacy of the combined MT technique compared with medium-bore DACs. By applying this technical modification, the rate of FPE was further increased, as the use of Catalyst 7 was the only predictor of FPE after multivariable analysis adjustment.

Catalyst 7 is currently the DAC with the largest distal inner diameter available that is fully compatible with the 
Table 3 Results of the multicollinearity test and univariable and multivariable logistic regression analyses for first-pass effect

\begin{tabular}{|c|c|c|c|c|}
\hline Variable & VIF & OR & $95 \% \mathrm{Cl}$ & $P$ value \\
\hline \multicolumn{5}{|l|}{ Univariable logistic regression analysis } \\
\hline Age (per 1 year increase) & 1.377 & 1.006 & 0.985 to 1.026 & 0.588 \\
\hline Initial CT ASPECTS (per 1-point increase) & 1.223 & 1.110 & 0.946 to 1.303 & $0.192^{*}$ \\
\hline Baseline NIHSS score (per 1-point increase) & 1.345 & 1.000 & 0.961 to 1.041 & 0.988 \\
\hline Onset to puncture time (per $1 \mathrm{~min}$ increase) & 1.237 & 0.999 & 0.997 to 1.001 & 0.302 \\
\hline Use of intravenous tPA & 1.269 & 1.199 & 0.706 to 2.037 & 0.501 \\
\hline Use of general anaesthesia & 1.795 & 0.834 & 0.494 to 1.409 & 0.497 \\
\hline Carotid T occlusion & 1.164 & 1.082 & 0.617 to 1.899 & 0.783 \\
\hline MCA M1 occlusion† & & 1.268 & 0.753 to 2.136 & 0.372 \\
\hline MCA M2 occlusion & 1.190 & 0.551 & 0.259 to 1.173 & $0.122^{*}$ \\
\hline Atherothrombotic origin & 1.287 & 0.631 & 0.274 to 1.452 & 0.279 \\
\hline Cardioembolic origin† & & 1.151 & 0.676 to 1.958 & 0.605 \\
\hline Other origin & 1.178 & 0.288 & 0.033 to 2.508 & 0.260 \\
\hline Undetermined origin & 1.265 & 1.227 & 0.677 to 2.225 & 0.501 \\
\hline Stroke centre $1 \dagger$ & & 1.215 & 0.717 to 2.059 & 0.470 \\
\hline Stroke centre 2 & 2.017 & 0.823 & 0.486 to 1.395 & 0.470 \\
\hline Use of Aperio & 1.880 & 0.495 & 0.228 to 1.078 & $0.077^{*}$ \\
\hline Use of Catch & 1.281 & 1.083 & 0.419 to 2.799 & 0.870 \\
\hline Use of Embotrap & 1.287 & 0.525 & 0.198 to 1.394 & $0.196^{\star}$ \\
\hline Use of pREset† & & 1.051 & 0.619 to 1.782 & 0.854 \\
\hline Use of Trevo & 2.115 & 1.801 & 1.012 to 3.205 & $0.046^{\star}$ \\
\hline Use of Catalyst $5 \dagger$ & & 0.577 & 0.325 to 1.025 & $0.061^{*}$ \\
\hline Use of Catalyst 6 & 2.299 & 0.698 & 0.398 to 1.224 & 0.210 \\
\hline Use of Catalyst 7 & 2.284 & 2.330 & 1.352 to 4.017 & $0.002^{*}$ \\
\hline \multicolumn{5}{|l|}{ Multivariable logistic regression analysis } \\
\hline Initial CT ASPECTS & & 1.169 & 0.985 to 1.388 & 0.074 \\
\hline MCA M2 occlusion & & 0.595 & 0.269 to 1.316 & 0.200 \\
\hline Use of Aperio & & 0.514 & 0.220 to 1.201 & 0.124 \\
\hline Use of Embotrap & & 0.420 & 0.141 to 1.253 & 0.120 \\
\hline Use of Trevo & & 1.383 & 0.729 to 2.623 & 0.321 \\
\hline Use of catalyst 5 & & 0.884 & 0.443 to 1.765 & 0.727 \\
\hline Use of catalyst 7 & & 2.336 & 1.191 to 4.581 & 0.014 \\
\hline
\end{tabular}

Numbers marked in bold indicate statistically significant differences $(P<0.05)$ in the multivariable analysis.

*Variables with $P<0.20$ in univariable analysis were included in the multivariable analysis.

†Independent variables excluded from multicollinearity test (predicted from one or more of the other independent variables).

ASPECTS, Alberta Stroke Program Early CT Score; eTICl, expanded thrombolysis in cerebral ischaemia; MCA, middle cerebral artery; NIHSS,

National Institutes of Health Stroke Scale; tPA, tissue-type plasminogen activator; VIF, variance inflation factor.

most widely used BGC, the FlowGate2. The suction force on the DAC tip is the product of the applied pressure and the cross-sectional area of the catheter; therefore, the catheter with the largest tip diameter will apply the greatest force. ${ }^{24}$ Since the distal inner diameters of Catalyst 5,6 and 7 are $1.47,1.52$ and $1.73 \mathrm{~mm}$, respectively, Catalyst 7 generates approximately $38 \%$ more aspiration force than Catalyst 5\% and 30\% higher than Catalyst 6 , which may explain its increased efficacy.

The performance of larger-diameter DACs in ADAPT has already been evaluated. The work of Delgado
Almandoz et $a l^{17}$ showed that the use of the large-bore ACE 68 (Penumbra, Alameda, California, USA) DAC was associated with shorter groin puncture to reperfusion time, higher rate of successful reperfusion and lower rate of stent retriever rescue compared with ACE 60 and ACE 64. Alawieh et al ${ }^{18}$ also compared multiple DACs with various inner diameters employing ADAPT, and although there were no differences in the reperfusion rates, they found a trend towards a gradual decrease in the use of rescue therapy and procedure times moving from smallest to largest catheter. However, these studies did not analyse 
FPE rates but rather employed the modified FPE (mTICI $2 \mathrm{~b} / 3$ after the first pass), and they did not fully provide the rate of failure to advance the DAC to the point of occlusion. One major concern about the use of large-bore DACs is navigability, especially in case of significant tortuosity in the carotid siphon. In our study, we managed to reach the thrombus with both the stent retriever and the DAC in all patients with the intention to apply PROTECTPLUS, with no differences between groups in terms of the type of arch or cervical or intracranial vascular tortuosity. One of the advantages of employing combined MT techniques is the possibility of using larger-calibre DACs, regardless of vascular tortuosity, since deploying the stent retriever serves as an anchor to advance the DAC more easily to the face of the thrombus.

A potential drawback of the combined use of largebore DACs with BGC is that the gap between the catheter lumen of the BGC and the aspiration catheter is too small, limiting the proximal aspiration force from the BGC. Nevertheless, it is at least possible to obtain local stasis in the ICA during the manoeuvre by inflating the balloon, and flow reversal is obtained immediately on withdrawal of the DAC and stent retriever to remove possible debris in the ICA and the risk of ENT.

By applying the larger-bore DAC, there was a significantly lower need of rescue therapy as well as a lower number of thrombectomy passes as compared with the medium-bore DACs. Concerning the latter point, recent data indicate that an increased number of thrombectomy attempts may have a negative impact on patients' clinical outcome. ${ }^{25}$ An advantage of fewer manoeuvres is that an increased number of passes can result in a higher rate of vessel dissections and haemorrhagic complications both by a potential perforation with the microwire or vessel stretching when performing an MT pass with stent retriever, which may explain the higher rate of subarachnoid haemorrhage in the Catalyst 5 group. In addition, a higher number of manoeuvres is associated with lengthy procedures, with a higher risk of microembolisms formation, which is another aspect that is not or not fully reflected in the current TICI-based grading scheme. In our study, we found shorter procedural times (from groin puncture to recanalisation) in the Catalyst 7 group compared with the Catalyst 5 group.

Concerning the safety outcomes, fewer overall procedure-related complications were encountered with the use of Catalyst 7, probably due to the higher aspiration force and the need for fewer thrombectomy passes. An important aspect addressed was the reduction of ENT, a complication consequence of thrombus fragmentation with the risk of occluding an artery that may be providing leptomeningeal collateral circulation to the ischaemic territory. Moreover, there is an added risk of producing new infarcts in a previously unaffected territory, with a significant worsening of the patient's clinical outcomes. ${ }^{26}$ Although the difference was not statistically significant, we found a tendency to a lower rate of ENT using Catalyst 7 compared with using the Catalyst 5 and 6 .
Recent evidence shows that improving the rate of complete first-pass reperfusion and decreasing the number of thrombectomy passes in total as well as the procedure time will ultimately result in clinical improvement and benefit for the patients. ${ }^{2352728}$ In our study, considering all patients treated with PROTECT-PLUS, the achievement of an FPE was associated with better clinical outcomes. Interestingly, although nearly all the aforementioned aspects of 'really successful' reperfusion were satisfactorily accomplished with the use of Catalyst 7 , no statistically significant differences between groups were observed in terms of functional independence at 3 months. However, there was a trend towards a higher rate of mRS score of 0-2 as the diameter of the DAC increased, $46 \%$ with Catalyst $5,50 \%$ with Catalyst $6 \%$ and $55 \%$ with Catalyst 7 . We believe that controlled clinical trials with a larger number of patients would more clearly address the clinical implications of using larger DACs in combined MT techniques.

Our work has limitations, which need to be considered when interpreting the results. First, as an observational retrospective study, it suffers from inherent methodological restrictions and a degree of selection bias may have been incurred; nonetheless, the study was conducted in two different comprehensive stroke centres and the cases included were consecutive patients with the intention to apply PROTECT-PLUS. We believe that the selection bias is of minor importance as the catheter selections were primarily driven by the availability in the department and not by the anatomy or vessel size. Furthermore, while both comprehensive stroke centres that included patients in this study carried out the same technique with the same main devices, there are intangible details regarding the working methods of both groups that could have influenced the results. In order to address this issue, we included the stroke centre as an independent variable in the logistic regression analysis. Finally, while the fact that different models and sizes of stent retrievers were employed should be mentioned as a possible bias, all stent retrievers were state-of-the-art devices with a balanced distribution of the type of stent retriever used among groups and also included in the logistic regression analysis.

\section{CONCLUSION}

In this study, the use of the larger-bore aspiration catheter as a further improvement of the combined MT technique led to a higher rate of FPE and higher reperfusion rates at the end of the procedure, a decreased number of thrombectomy attempts, shorter procedural times and lower need of rescue therapy while reducing the overall procedure-related complications compared with the use of medium-bore aspiration catheters.

Contributors Protocol/project development and interventional procedures performance: MM, CM, CP-G, CZ, MTB, CR and SR. Data collection and management: CP-G, CM, MM, SR, CZ, CG-E, MTB and CR. Manuscript writing/ editing and critical revision of the work for important intellectual content: $\mathrm{CP}-\mathrm{G}$, 
$\mathrm{CM}, \mathrm{CZ}, \mathrm{MTB}, \mathrm{CR}, \mathrm{MM}$ and JA. Final approval of the manuscript and agreement to be accountable for all aspects of the work: CP-G, CM, MM, SR, CZ, CG-E, MTB, JA and $\mathrm{CR}$.

Funding The authors have not declared a specific grant for this research from any funding agency in the public, commercial or not-for-profit sectors.

Competing interests None declared.

Patient consent for publication Not required.

Ethics approval All procedures performed in the study involving human participants were in accordance with the 1964 Helsinki Declaration and its later amendments or comparable ethical standards. The Hospital Clínico Universitario San Carlos ethics committee gave the project a positive vote under number 20/700-E and the need for patient consent was waived.

Provenance and peer review Not commissioned; externally peer reviewed. Data availability statement Data are available upon reasonable request. The data that support the findings of this study are available from the corresponding author upon reasonable request.

Open access This is an open access article distributed in accordance with the Creative Commons Attribution Non Commercial (CC BY-NC 4.0) license, which permits others to distribute, remix, adapt, build upon this work non-commercially, and license their derivative works on different terms, provided the original work is properly cited, appropriate credit is given, any changes made indicated, and the use is non-commercial. See: http://creativecommons.org/licenses/by-nc/4.0/.

ORCID iD

Carlos Pérez-García http://orcid.org/0000-0002-5450-148X

\section{REFERENCES}

1 Goyal M, Menon BK, van Zwam WH, et al. Endovascular thrombectomy after large-vessel ischaemic stroke: a meta-analysis of individual patient data from five randomised trials. Lancet 2016;387:1723-31.

2 Kaesmacher J, Maegerlein C, Zibold F, et al. Improving mTICI2b reperfusion to $\mathrm{mTICl} 2 \mathrm{c} / 3$ reperfusions: a retrospective observational study assessing technical feasibility, safety and clinical efficacy. Eur Radiol 2018;28:274-82.

3 Kleine JF, Wunderlich S, Zimmer C, et al. Time to redefine success? $\mathrm{TICl} 3$ versus $\mathrm{TICI} 2 \mathrm{~B}$ recanalization in middle cerebral artery occlusion treated with thrombectomy. J Neurointerv Surg 2017;9:117-21.

4 Mokin M, Primiani CT, Castonguay AC, et al. First pass effect in patients treated with the Trevo Stent-Retriever: a track registry study analysis. Front Neurol 2020;11:1-6.

5 Nikoubashman O, Dekeyzer S, Riabikin A. True first-pass effect: first-pass complete reperfusion improves clinical outcome in thrombectomy stroke patients. Stroke 2019;50:2140-6.

6 Velasco A, Buerke B, Stracke CP, et al. Comparison of a balloon guide catheter and a Non-Balloon guide catheter for mechanical thrombectomy. Radiology 2016;280:169-76

7 Brinjikji W, Starke RM, Murad MH, et al. Impact of balloon guide catheter on technical and clinical outcomes: a systematic review and meta-analysis. J Neurointerv Surg 2018;10:335-9.

8 Nguyen TN, Malisch T, Castonguay AC. Balloon guide catheter improves revascularization and clinical outcomes with the solitaire device : Analysis of the north american solitaire acute stroke registry. Stroke 2014:45:141-5

9 Kim Y-W, Kang D-H, Hwang Y-H, et al. Efficacy of proximal aspiration thrombectomy for using balloon-tipped guide catheter in acute intracranial internal carotid artery occlusion. $J$ Korean Neurosurg Soc 2016:59:379-84.

10 Stampfl S, Pfaff J, Herweh C, et al. Combined proximal balloon occlusion and distal aspiration: a new approach to prevent distal embolization during neurothrombectomy. J Neurointerv Surg 2017;9:346-51.

11 Lapergue B, Blanc R, Gory B, et al. Effect of endovascular contact aspiration vs stent retriever on revascularization in patients with acute ischemic stroke and large vessel occlusion. JAMA 2017;318:443-52.

12 Lapergue B, Blanc R, Guedin P, et al. A direct aspiration, first pass technique (adapt) versus stent retrievers for acute stroke therapy: an observational comparative study. AJNR Am J Neuroradiol 2016;37:1860-5.

13 Maus V, Behme D, Kabbasch C, et al. Maximizing first-pass complete reperfusion with save. Clin Neuroradiol 2018;28:327-38.

14 McTaggart RA, Tung EL, Yaghi S, et al. Continuous aspiration prior to intracranial vascular embolectomy (captive): a technique which improves outcomes. J Neurointerv Surg 2017;9:1154-9.

15 Maegerlein C, Mönch S, Boeckh-Behrens T, et al. Protect: proximal balloon occlusion together with direCt thrombus aspiration during stent retriever thrombectomy - evaluation of a double embolic protection approach in endovascular stroke treatment. J Neurointerv Surg 2018;10:751-5.

16 Maegerlein C, Berndt MT, Mönch S, et al. Further development of combined techniques using stent Retrievers, aspiration catheters and BGC. Clin Neuroradiol 2020;30:59-65.

17 Delgado Almandoz JE, Kayan Y, Wallace AN, et al. Larger ACE 68 aspiration catheter increases first-pass efficacy of adapt technique. $J$ Neurointerv Surg 2019;11:141-6.

18 Alawieh A, Chatterjee AR, Vargas J, et al. Lessons learned over more than 500 stroke Thrombectomies using adapt with increasing aspiration catheter size. Neurosurgery 2020;86:61-9.

19 Haussen DC, Rebello LC, Nogueira RG. Optimizating clot retrieval in acute stroke: the push and fluff technique for closed-cell stentrievers. Stroke 2015;46:2838-42.

20 Madhwal S, Rajagopal V, Bhatt DL, et al. Predictors of difficult carotid stenting as determined by aortic arch angiography. J Invasive Cardiol 2008;20:200-4.

21 Nagata T, Masumoto K, Hayashi Y, et al. Three-dimensional computed tomographic analysis of variations of the carotid artery. Journal of Cranio-Maxillofacial Surgery 2016;44:734-42.

22 Lin L-M, Colby GP, Jiang B, et al. Classification of cavernous internal carotid artery tortuosity: a predictor of procedural complexity in pipeline embolization. J Neurointerv Surg 2015;7:628-33.

23 Adams HP, Bendixen BH, Kappelle LJ, et al. Classification of subtype of acute ischemic stroke. definitions for use in a multicenter clinical trial. TOAST. trial of ORG 10172 in acute stroke treatment. Stroke 1993;24:35-41.

24 Nikoubashman O, Nikoubashman A, Büsen M, et al. Necessary catheter diameters for mechanical thrombectomy with adapt. AJNR Am J Neuroradiol 2017;38:2277-81.

25 Ozdemir O, Giray S, Arlier Z, et al. Predictors of a good outcome after endovascular stroke treatment with stent retrievers. ScientificWorldJournal 2015;2015:1-9.

26 Goyal M, Menon BK, Demchuk A, et al. Proposed methodology and classification of infarct in new Territory (int) after endovascular stroke treatment. J Neurointerv Surg 2017;9:449-50.

27 McTaggart RA, Ospel JM, Psychogios M-N, et al. Optimization of endovascular therapy in the Neuroangiography suite to achieve fast and complete (expanded treatment in cerebral ischemia 2c-3) reperfusion. Stroke 2020;51:1961-8.

28 Zaidat OO, Castonguay AC, Linfante I, et al. First pass effect: a new measure for stroke thrombectomy devices. Stroke 2018;49:660-6. 